\title{
Myzus persicae (Sulzer) in Puerto Rico
}

\author{
Clyde F. Smith ${ }^{1}$, L. F. Martorell ${ }^{2}$, and M. E. Pérez-Escolar ${ }^{3}$
}

\section{INTRODUCTION}

The green peach aphid, Myzus persicae (Sulzer) has been recorded as a pest of tobacco in Puerto Rico since $1950-51$, Wolcott (5) ${ }^{4}$; however, it has been reported since 1916, Wolcott (B), on the following host plants: Eggplant, pepper, potato, tomato, sweetpotato, and sesame. Lately, it has been observed by Adsuar, Wolcott, and Martorell on ground cherry, Physalis angulata L., wire-weed, Sida carpinifolia L. f., and papaya, Carica papaya $\mathrm{L}$.

In 1954 and again in 1955, the senior author was invited to Puerto Rico to make a survey of the distribution and host plants of the peach aphid. The survey was made during the season when tobacco was not being grown, July and August 1954, and just as the crop was being harvested, February and March 1955. Martorell and Pérez made additional observations and collections during the interim.

\section{RESULTS OF SURVEY}

During the "off-season", July and August, Myzus persicae was commonly found on peppers which were being grown in home gardens and commercial gardens. This is significant because peppers are known to harbor one important disease of tobacco in Puerto Rico, namely the pepper-mosaic virus. Thus the pepper plants may serve as a reservoir for the disease and also for the aphids. This disease has been studied previously by Roque and Adsuar (2) and lately by Pérez, Adsuar, and Padilla (1). The virus causing the mosaic disease of peppers in Puerto Rico is transmitted from pepper to tobacco and vice versa by the common green peach aphid. This virus is also transmitted mechanically. Another common virus of tobacco in Puerto Rico is transmitted to peppers and from peppers again to tobacco by mechanical means and not by any type of insect vector. A full account of these pepper and tobacco diseases is given in the publications mentioned above.

Several other plants also serve as alternate hosts (see host list, table 1) and all of them could play an important role as a source of infestation for

${ }^{1}$ Head, Department of Entomology, North Carolina State College, Raleigh, N. C.

${ }^{2}$ Head, Department of Entomology, Agricultural Experiment Station, University of Puerto Rico, Río Piedras, P.R.

${ }^{3}$ Assistant Entomologist, Agricultural Experiment Station, University of Puerto Rico, Río Piedras, P.R.

Italic letters in parentheses refer to Literature Cited, p. 266. 
new tobacco. However, observations by Perez indicate that sweetpotatoes may be one of the most important sources of infestation for tobacco because the sweetpotato is very common in Puerto Rico, being grown in rotation with tobacco or in the vicinity of tobacco, and it usually has a very heavy infestation of Myzus persicae just at the time tobacco is being set into the field. Young sweetpotato shoots are also commonly infested with aphids and are quite common in fields that have been harvested, thus creating

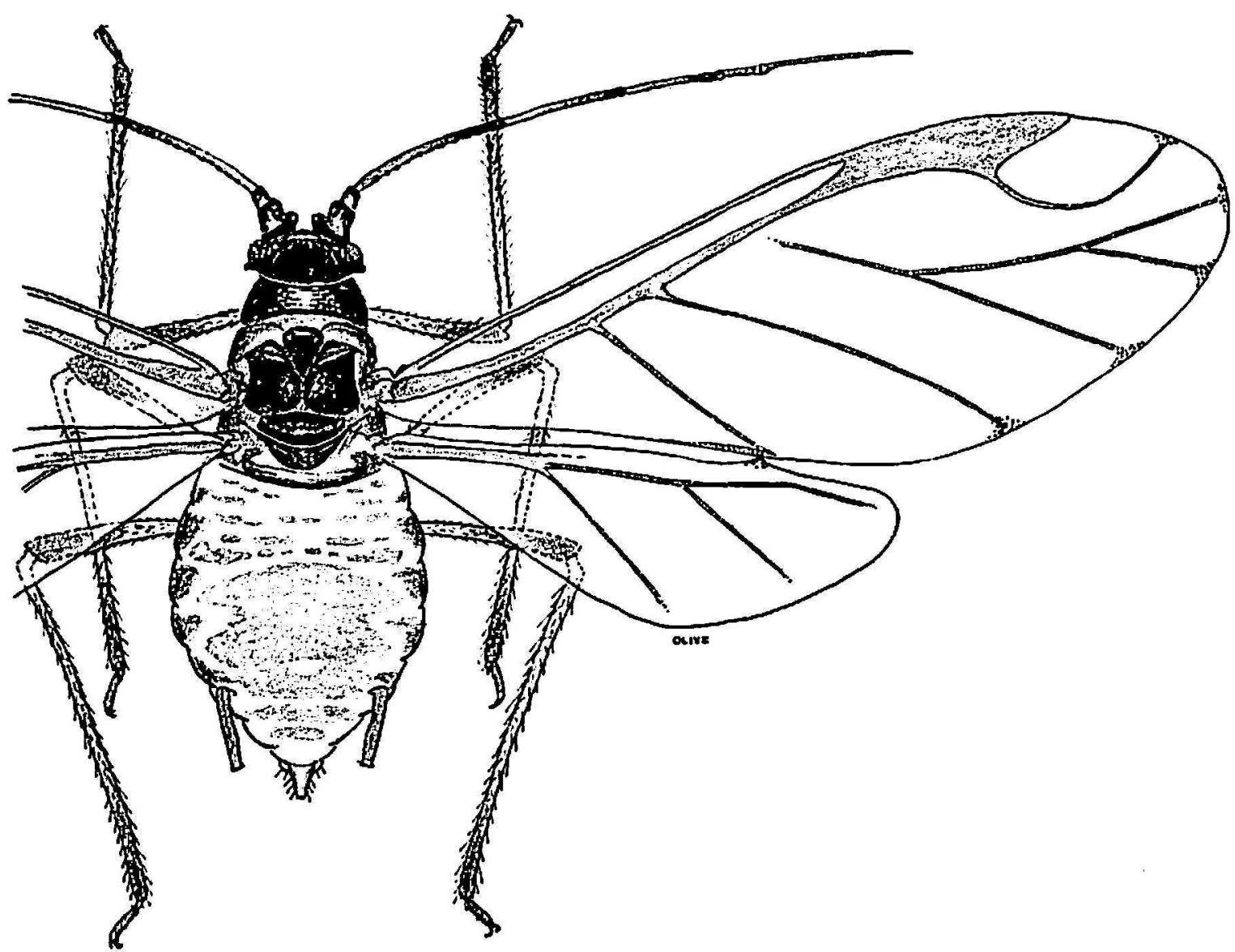

Fra. 1.-Winged alate of Myzus persicac (Sulzer). Drawing by Tom Olive, North Carolina State College, Department of Entomology.

an ideal source of infestation. In general the infestation does not occur in the plant bed as is often common in the United States but usually occurs after the plants are set in the field.

Myzus persicae (fig. 1) is common and often occurs in damaging numbers on tobacco in all areas of Puerto Rico where tobacco is grown. Table 1 is a list of the other hosts upon which $M$. persicae has been collected at various locations in Puerto Rico, and one in Haiti.

\section{SUMMARY}

The green peach aphid, Myzus persicae Sulzer, is a pest of tobacco in l'uerto Rico. This insect is also a vector of virus diseases transmitted from 
TABLE 1.-Host list for Myzus persicae

\begin{tabular}{|c|c|c|}
\hline Host & Location & Date \\
\hline $\begin{array}{l}\text { Apium graveolens } \\
\text { Borreria verticillata }\end{array}$ & $\begin{array}{l}\text { Cayey } \\
\text { Guajataca Gorge, Quebradi- } \\
\text { llas }\end{array}$ & $\begin{array}{l}\text { Mar. 8, } 1955 \\
\text { Mar. 1, } 1955\end{array}$ \\
\hline Brassica integrifolia & $\begin{array}{l}\text { Cayey } \\
\text { Cayey-Cidra Road } \\
\text { Río Piedras }\end{array}$ & $\begin{array}{l}\text { Oct. 26, } 1954 \\
\text { Nov. 16, } 1954 \\
\text { Nov. 22, 1954 } \\
\text { Mar. 8, } 1955 \\
\text { Sept. 19, 1954 } \\
\text { Sept. 22, 1954 } \\
\text { Sept. 28, 1954 } \\
\text { Mar. 7, 1954 }\end{array}$ \\
\hline $\begin{array}{l}\text { B. oleracea } \\
\text { Capsicum sp. }\end{array}$ & Cayey & $\begin{array}{l}\text { Mar. } 8,1955 \\
\text { Aug. } 1,1954\end{array}$ \\
\hline capsictin sp. & $\begin{array}{l}\text { Cayey-Cidra Road } \\
\text { Rammey Field, Aguadilla }\end{array}$ & $\begin{array}{l}\text { Aug. 2, } 1954 \\
\text { Sept. 22, } 1954 \\
\text { July 21, } 1954\end{array}$ \\
\hline C. annuum & $\begin{array}{l}\text { Cayey } \\
\text { Río Piedras }\end{array}$ & $\begin{array}{l}\text { Mar. 8, } 1955 \\
\text { Mar. 7, } 1955\end{array}$ \\
\hline $\begin{array}{l}\text { Chenopodium ambrosioides } \\
\text { Cleome gynandra }\end{array}$ & $\begin{array}{l}\text { Barrio Rincón, Cidra } \\
\text { Cayey } \\
\text { Isabela }\end{array}$ & $\begin{array}{l}\text { Feb. } 28,1955 \\
\text { Oct. } 19,1954 \\
\text { Apr. } 1,1955\end{array}$ \\
\hline $\begin{array}{l}\text { Clerodendron fragans } \\
\text { Emilia coccinea }\end{array}$ & Cayey & $\begin{array}{l}\text { Mar. 8, } 1955 \\
\text { Mar. } 16,1955 \\
\text { Mar } 16,1955\end{array}$ \\
\hline E. sonchifolia & Cidra & Sept. 28, 1954 \\
\hline $\begin{array}{l}\text { Fleurya aestuans } \\
\text { Ipomoea batalas }\end{array}$ & $\begin{array}{l}\text { Cayey } \\
\text { Treasure Island, Cidra } \\
\text { Cayey-Caguas Road } \\
\text { Cidra }\end{array}$ & $\begin{array}{l}\text { Mar. 8, } 1955 \\
\text { Feb. 28, } 1955 \\
\text { Mar. 16, } 1955 \\
\text { Oct. } 5,1954 \\
\text { Oct. } 10,1954\end{array}$ \\
\hline $\begin{array}{l}\text { Lactuca floridana } \\
L \text { saliva } \\
\text { Moringa oleifera } \\
\text { Nicotiana tabacum }\end{array}$ & $\begin{array}{l}\text { Ciales-Jayuya Road } \\
\text { Rammey Field, Aguadilla } \\
\text { Sabana Grande-Yauco Road } \\
\text { Cayey-Caguas Road } \\
\text { Rí Piedras-Caguas Road } \\
\text { Cidra } \\
\text { Sabana Grande-Yauco Road } \\
\text { Cidra } \\
\text { Rio Piedras } \\
\text { Cayey-Salinas Road } \\
\text { Isabela } \\
\text { Cayey }\end{array}$ & $\begin{array}{l}\text { Mar. 2, 1955 } \\
\text { July 21, 1954 } \\
\text { Mar. 11, } 1955 \\
\text { Mar. 16, } 1955 \\
\text { Feb. 28, 1955 } \\
\text { Nov. 9, } 1954 \\
\text { Mar. 11, 1955 } \\
\text { Dec. 17, 1954 } \\
\text { Nov. 6, 1954 } \\
\text { Dec. 17, } 1954 \\
\text { Dec. 28, 1954 } \\
\text { Mar. 18, } 1955\end{array}$ \\
\hline $\begin{array}{l}\text { Physalis pubescens } \\
\text { P. turbinata } \\
\text { Polianthes tuberosa } \\
\text { Polyscias quilfoylei } \\
\text { Senecio confusus } \\
\text { Solanum melongena } \\
\text { S. nigrum } \\
\text { Spondias purpurea } \\
\text { Tectona grandis } \\
\text { B integrifolia }\end{array}$ & $\begin{array}{l}\text { El Collao-Cayey Road } \\
\text { Cayey-Caguas Road } \\
\text { Río Piedras } \\
\text { Río Grande } \\
\text { Ciales-Jayuya Road } \\
\text { Río Piedras } \\
\text { Cayey-Caguas Road } \\
\text { Cayey-Salinas Road } \\
\text { Cayey } \\
\text { Chalet de Felurs, Haiti } \\
\text { Cayey }\end{array}$ & $\begin{array}{l}\text { Mar. } 11,1955 \\
\text { Mar. 16, } 1955 \\
\text { Mar. 4, } 1955 \\
\text { Mar. 9, } 1955 \\
\text { Mar. 2, 1955 } \\
\text { Mar. 7, } 1955 \\
\text { Mar. 16, } 1955 \\
\text { Feb. 28, 1955 } \\
\text { Mar. 8, } 1955 \\
\text { Feb. 25, 1955 } \\
\text { Oct. 19, } 1954 \\
\text { Dec. 17. } 1954\end{array}$ \\
\hline Unidentified crucifcrous plant & Lajas Substation & Aug. 12, 1954 \\
\hline
\end{tabular}


pepper to tobacco and vice-versa. During a survey made in Puerto Rico by the authors, the known host-plant list of this insect was greatly increased by adding around 15 new records.

\section{RESUMEN}

El pulgón verde, Myzus persicae Sulzer, es una plaga del tabaco en Puerto Rico. Este insecto es también el vector de enfermedades virosas trasmitidas del pimiento al tabaco y viceversa. Durante un catastro efectuado en la Isla por los autores, la lista ya conocida de plantas hospedadoras de esta especie se aumentó al añadirle alrededor de 15 nuevos récords.

\section{LITERATURE CITED}

1. Pérez, Juan E., and Adsuar, José, A survey for common tobacco- and peppermosaic viruses in the filler-tobacco area of Puerto Rico, J. Agr. Univ. P.R. 41 (1) $20-4,1957$.

2. Roque, Arturo, and Adsuar, Jose, Studies on the mosaic of peppers, (Capsicum frutescens) in Puerto Rico, J. Agr. Univ. P.R. 25 (3) 40-50, 1941.

3. Wolcott, G. N., Insectae Borinquenses, J. Agr. Univ. P.R., 20 (1-4) 118, 1936.

4. - The insects of Puerto Rico, J. Agr. Univ. P.R. 32 (1) 155-6, 1948.

5. —, Migrating Aphids, Sci. 116 (3002) 43-4, ref. 5, Lancaster, Pa. July 11, 1952. 